\title{
AREA OF FATOU SETS OF TRIGONOMETRIC FUNCTIONS
}

\author{
HENDRIK SCHUBERT
}

(Communicated by Juha M. Heinonen)

\begin{abstract}
We extend a result of McMullen to show that the area of the Fatou set of the sine function in a vertical strip of width $2 \pi$ is finite. This confirms a conjecture by Milnor.
\end{abstract}

\section{INTRODUCTION}

Let $f$ denote a transcendental entire function of one complex variable and $f^{n}$, $n \in \mathbb{N}$, the $n$th iterate. The Fatou set of $f$, denoted by $F(f)$, is defined as the set of points $z \in \mathbb{C}$ so that the sequence $\left(f^{n}\right)_{n \in \mathbb{N}}$ forms a normal family in a neighbourhood of $z$. It is easy to see that $F(f)$ is an open set, and $F(f)$ is completely invariant under $f$. The complement of $F(f)$ is the Julia set $J(f)$ of $f$. This set is obviously closed and also completely invariant under $f$. For more detail about these sets, see, e.g., $[1,10,14]$. These authors consider only rational functions, but the concepts remain the same for transcendental functions [2].

Apart from the exponential family, the transcendental entire functions most studied in complex dynamics are the trigonometric functions. Already Pierre Fatou [6] considered iteration of functions of the form $f(z)=h \sin (z)+a, 0<h<1, a \in \mathbb{R}$. Hans Töpfer [15] studied the dynamics of $\sin (z)$ and $\cos (z)$ a few years later. More recent work begins with a paper by Curt McMullen [9] who showed that the Julia set of any function of the form $f(z)=\sin (\alpha z+\beta), \alpha \neq 0$ has positive area. Since then a number of papers concerning the dynamics of trigonometric functions have appeared where various aspects were discussed, e.g. connectedness properties and buried points of the Julia set of $\sin (z)$ by Patricia Domínguez [3], the dynamics of $\lambda \sin (z)$ by Patricia Domínguez and Guillermo Sienra [4], the set of accessible points of the Julia set of $\lambda \sin (z)$ by Boguslawa Karpinska [7, 8], the dynamical fine structure of iterated cosine maps by Dierk Schleicher [12] or the set of escaping points of the cosine family by Günther Rottenfußer and Dierk Schleicher [11].

John Milnor conjectured in $[10$, p. 64] that the Fatou set of the sine function has finite area in a vertical strip of width $2 \pi$. In this article we prove his conjecture following the ideas of McMullen's proof in [9]. As we use some of his lemmas, we will not work with the sine function but with the hyperbolic sine. Note that $\sinh (z)=$ $-i \sin (i z)$ so that Milnor's conjecture is equivalent to the following theorem.

Theorem 1.1. The area of the Fatou set of the hyperbolic sine is finite in a horizontal strip of width $2 \pi$.

Received by the editors August 11, 2004 and, in revised form, November 20, 2006.

2000 Mathematics Subject Classification. Primary 37F10; Secondary 30D05. 
As in [9] we consider the set $I(\sinh )$ of points that tend to $\infty$ under iteration. It was shown by McMullen in [9] and also follows from a more general result of Eremenko and Lyubich [5] that $I(\sinh ) \subset J(\sinh )$. Thus Theorem 1.1 follows directly from the next theorem.

Theorem 1.2. Let $S$ be a horizontal strip of width $2 \pi$. Then $S \backslash I(\sinh )$ has finite area.

These theorems are the main result of my diploma thesis [13].

\section{Distortion AND NONLINEARITY}

Let $D$ be a bounded subset of the complex plane and $f$ a map which is holomorphic in a neighbourhood of $D$. We say that $f$ has bounded distortion on $D$ if there are constants $c, C>0$ with

$$
c<\frac{|f(x)-f(y)|}{|x-y|}<C
$$

for all $x, y \in D$ with $x \neq y$.

We call

$$
L\left(f_{\mid D}\right):=\inf \{C / c:(c, C) \text { satisfies }(1)\}
$$

the distortion of $f$ on $D$. If $L\left(f_{\mid D}\right)$ is close to one, we say that $f$ has small distortion on $D$. Small distortion implies that $f$ nearly preserves relative lengths and measures. We define

$$
\operatorname{dens}(A, D):=\frac{\operatorname{meas}(A \cap D)}{\operatorname{meas}(D)}
$$

for two measurable subsets $A$ and $D$ of $\mathbb{C}$, where meas denotes the plane Lebesgue measure. We call dens $(A, D)$ the density of $A$ in $D$.

It is clear that

$$
\text { dens }(f(A), f(D)) \leq L\left(f_{\mid D}\right)^{2} \operatorname{dens}(A, D),
$$

and it is straightforward to show that the distortion of holomorphic functions satisfies

$$
\begin{gathered}
L\left(f_{\mid D}\right)=L\left(f_{\mid f(D)}^{-1}\right), \\
L\left((g \circ f)_{\mid D}\right) \leq L\left(f_{\mid D}\right) L\left(g_{\mid f(D)}\right),
\end{gathered}
$$

and

$$
L\left(f_{\mid D}\right) \geq \frac{\sup _{z \in D}\left|f^{\prime}(z)\right|}{\inf _{z \in D}\left|f^{\prime}(z)\right|} .
$$

For a conformal map $f$ a differential quantity related to the distortion is the nonlinearity of $f$. Let $D$ be a bounded subset of the complex plane and let $f$ be a conformal map on $D$. We define the nonlinearity of $f$ on $D$ as

$$
N\left(f_{\mid D}\right):=\sup \left\{\frac{\left|f^{\prime \prime}(z)\right|}{\left|f^{\prime}(z)\right|}: z \in D\right\} \operatorname{diam}(D),
$$

provided the right-hand side is finite. This is always the case if $D$ is compact and $f$ is conformal in a neighbourhood of $D$. Later we will estimate the distortion of a function on squares. Here and in the following by square we mean a closed square whose sides are parallel to the coordinate axes. The relation between the distortion and nonlinearity on squares follows with the next lemma. 
Lemma 2.1. Let $Q$ be a square and let $f$ be a conformal map defined in a neighbourhood of $Q$ with $N\left(f_{\mid Q}\right)<1 / 4$. Then

$$
L\left(f_{\mid Q}\right) \leq 1+8 N\left(f_{\mid Q}\right) .
$$

Remark. Lemma 2.1 is due to McMullen [9]. He only notes that $L\left(f_{\mid Q}\right)$ is bounded by $1+O\left(N\left(f_{\mid Q}\right)\right)$, when $N\left(f_{\mid Q}\right)$ is near 0 , but a computation yields the explicit constants given above.

The next lemma allows us in some cases to control the distortion of a function over any number of iterates.

Lemma 2.2. Let $n \in \mathbb{N}$. For all $i \in \mathbb{N}$ with $i \leq n$ let $D_{i} \subset \mathbb{C}$ be open sets and let $f_{i}: D_{i} \rightarrow \mathbb{C}$ be conformal maps. Let $\alpha, M>0$ be constants with

$$
\left|f_{i}^{\prime}(z)\right|>\alpha>1 \text { and } \frac{\left|f_{i}^{\prime \prime}(z)\right|}{\left|f_{i}^{\prime}(z)\right|}<M
$$

for all $i \in \mathbb{N}$ with $i \leq n$ and $z \in D_{i}$. Furthermore, for each $i \in \mathbb{N}$ with $i \leq n$, let $Q_{i} \subset D_{i}$ be a square with sides of length $r$ satisfying $Q_{i+1} \subset f_{i}\left(Q_{i}\right)$. Define $D:=f_{n}\left(Q_{n}\right)$ and

$$
h: D \rightarrow Q_{1}, z \mapsto\left(f_{n} \circ \ldots \circ f_{1}\right)^{-1}(z) .
$$

Then $h$ is a conformal map and there is a constant $c_{1}>0$, depending on $\alpha, M$ and $r$ but not on $n$, with

$$
L\left(h_{\mid D}\right)<c_{1} .
$$

Remark. Lemma 2.2 is proved by McMullen [9]. A computation shows that if we take $r:=1 /(8 \sqrt{2} M)$, then the quantities $r$ and $M$ cancel each other and we get

$$
c_{1}:=2 \exp \left(\frac{1}{1-1 / \alpha}\right) \text {. }
$$

For the next lemma we need some definitions. For all $x>0$, we define

$$
R(x):=\{z \in \mathbb{C}:|\operatorname{Re} z| \geq x\}
$$

and for all $r>0$ we denote by $G_{r}$ the set of squares $Q_{r}$ of $\mathbb{C}$ consisting of points $z \in \mathbb{C}$ for which $n r \leq \operatorname{Re} z \leq(n+1) r$ and $m r \leq \operatorname{Im} z \leq(m+1) r$ for some $m, n \in \mathbb{Z}$. We call $G_{r}$ a grid of length $r$.

Lemma 2.3. Let $x>0$ and $Q \subset \mathbb{C}$ be a square with sides of length $r>0$. Let $z_{0} \in Q$ and $f$ be a conformal map which is defined in a neighbourhood of $Q$ and has bounded distortion on $Q$. Then

$\#\left\{Q_{r} \in G_{r}: Q_{r} \cap \partial f(Q) \neq \emptyset\right.$ or $\left.Q_{r} \cap(\partial R(x) \cap f(Q)) \neq \emptyset\right\} \leq 12+c_{2} L\left(f_{\mid Q}\right)\left|f^{\prime}\left(z_{0}\right)\right|$ where $c_{2}:=3(4+2 \sqrt{2})$.

Proof. First we note that if $\gamma \subset \mathbb{C}$ is a curve of length $l$, then

$$
\#\left\{Q_{r} \in G_{r}: Q_{r} \cap \gamma \neq \emptyset\right\} \leq 4+\frac{3 l}{r} .
$$

To see this, take a curve of length $r$ with starting point $s$. Then $s$ can intersect at most four squares. And besides the square(s) which is (are) intersected by $s$, the curve can intersect at most three other squares. Dividing $\gamma$ in parts of length $r$ yields (7). Let $l_{1}$ be the length of $\partial f(Q)$. Using the relation $\sup _{z \in Q}\left|f^{\prime}(z)\right| \leq L\left(f_{\mid Q}\right)\left|f^{\prime}\left(z_{0}\right)\right|$ it is easy to see that

$$
l_{1} \leq 4 L\left(f_{\mid Q}\right)\left|f^{\prime}\left(z_{0}\right)\right| r .
$$


As $\partial R(x) \cap f(Q) \cap\{z \in \mathbb{C}: \operatorname{Re} z>0\}$ consists of segments of a straight line with length

$$
l_{2} \leq \operatorname{diam} f(Q) \leq \sqrt{2} L\left(f_{\mid Q}\right)\left|f^{\prime}\left(z_{0}\right)\right| r,
$$

it follows from (7) that

$$
\#\left\{Q_{r} \in G_{r}: Q_{r} \cap \partial f(Q) \neq \emptyset \text { or } Q_{r} \cap(\partial R(x) \cap f(Q)) \neq \emptyset\right\} \leq 12+\frac{3\left(l_{1}+2 l_{2}\right)}{r} .
$$

This completes the proof.

\section{Nested SETS}

In order to get an estimation of the measure of $I(f)$, we will construct subsets by nested intersection of dynamically defined sets. A general estimation for the measure of the resulting sets is given by Lemma 3.2 below.

Definition 3.1 (Nesting conditions). For all $k \in \mathbb{N}_{0}$ let $E_{k}$ be a finite collection of measureable subsets of $\mathbb{C}$, i.e. $E_{k}:=\left\{F_{k, i} \subset \mathbb{C}: i \in\left\{1, \ldots, l_{k}\right\}\right\}$ with $l_{k}:=\# E_{k} \in$ $\mathbb{N}_{0}$. Then the sequence $\left(E_{k}\right)_{k \in \mathbb{N}_{0}}$ satisfies the nesting conditions if $E_{0}=\left\{F_{0,1}\right\}$, where $F_{0,1}$ is a compact connected set and for all $k \in \mathbb{N}_{0}$

for all $i \in\left\{1, \ldots, l_{k+1}\right\}$ there is $j \in\left\{1, \ldots, l_{k}\right\}$ with

$$
F_{k+1, i} \subset F_{k, j},
$$

for all $i \in\left\{1, \ldots, l_{k}\right\}$ there is $j \in\left\{1, \ldots, l_{k+1}\right\}$ with

$$
F_{k+1, j} \subset F_{k, i},
$$

for all $i, i^{\prime} \in\left\{1, \ldots, l_{k}\right\}$ with $i \neq i^{\prime}$ we have

$$
\operatorname{meas}\left(F_{k, i} \cap F_{k, i^{\prime}}\right)=0 \text {, }
$$

there is $\delta_{k}>0$ such that for all $i \in\left\{1, \ldots, l_{k}\right\}$ and $F_{k, i} \in E_{k}$ we have

$$
\operatorname{dens}\left(\bigcup_{j=1}^{l_{k+1}} F_{k+1, j}, F_{k, i}\right) \geq \delta_{k} .
$$

Define $E:=\bigcap_{k=1}^{\infty}\left(\bigcup_{i=1}^{l_{k}} F_{k, i}\right)$. The following result is due to McMullen [9].

Lemma 3.2. Let $\left(E_{k}\right)_{k \in \mathbb{N}_{0}}$ be a sequence that satisfies the nesting conditions. Then

$$
\text { dens }\left(E, F_{0,1}\right) \geq \prod_{k=0}^{\infty} \delta_{k} \text {. }
$$

\section{Proof of Theorem 1.2}

From now on the function $f$ will be the hyperbolic sine; i.e.

$$
f: \mathbb{C} \rightarrow \mathbb{C}, z \mapsto \frac{1}{2}\left(e^{z}-e^{-z}\right) .
$$

Outline of the proof. The most important step in the proof is to get an estimate of the density of $I(f)$ in a square depending on the location of the square in the complex plane. Therefore, we take a square lying away from the imaginary axis and look for points in this square which tend very rapidly to infinity under iteration. We take a real sequence $\left(x_{k}\right)_{k \in \mathbb{N}_{0}}$ which converges to infinity and define the following collection of subsets of $\mathbb{C}$ inductively. Let $E_{0}=\left\{Q^{*}\right\}$ where $Q^{*}$ is a square in $R\left(x_{0}\right)$ 
with sides of length $r$. Furthermore, let $E_{k}$ be the set consisting of those subsets of $Q^{*}$ for which the image under $f^{k}$ is an element of the grid $G_{r}$ which lies in the intersection of $R\left(x_{k}\right)$ and the image of an element of $E_{k-1}$ under $f^{k}$. Thus points in $\bigcap_{k=0}^{\infty} E_{k}$ converge to infinity under iteration and, therefore, lie in $I(f)$. In order to apply Lemma 3.2, we have to show that the nesting conditions in Definition 3.1 are satisfied. Because of the construction it will be easy to see that the nesting conditions (8), (9) and (10) hold, but (11) is more delicate. Using Lemmas 2.1 and 2.3 , it is possible to show that for any square $Q$ in $R\left(x_{k}\right)$ with sides of length $r$, the union of the elements of $G_{r}$ that lie in the intersection of $R\left(x_{k+1}\right)$ and $f(Q)$ has a density in $f(Q)$ which is larger than $1-O\left(x_{k+1} / \exp \left(x_{k}\right)\right)$. The distortion Lemma 2.2 and inequality (2) then yield (11) with $\delta_{k}=1-O\left(x_{k+1} / \exp \left(x_{k}\right)\right)$. Applying Lemma 3.2 we get $\operatorname{dens}\left(I(f), Q^{*}\right)>1-O(1 / \exp (x / 2))$ for all squares $Q^{*}$ in $R(x)$ as $x \rightarrow \infty$ and from here the claim follows.

Let $x^{*}>0$ with

$$
x^{*}>4+2 c_{2} \text {. }
$$

It is elementary that for all $z \in R\left(x^{*}\right)$

$$
\left|f^{\prime}(z)\right|>\frac{1}{4} \exp (|\operatorname{Re} z|)>2
$$

and

$$
\left|\frac{f^{\prime \prime}(z)}{f^{\prime}(z)}\right|<2
$$

Lemma 4.1. Let $x \geq x^{*}$ and define $x_{k}:=2 \exp ^{k}(x / 2)$ for all $k \in \mathbb{N}_{0}$. Then there is a constant $c_{3}>0$ such that if $Q \subset R\left(x_{k}\right)$ is a square with sides of length $r=1 / 16 \sqrt{2}$, then

$$
\operatorname{dens}\left(\bigcup\left\{Q_{r} \in G_{r}: Q_{r} \subset f(Q) \cap R\left(x_{k+1}\right)\right\}, f(Q)\right) \geq 1-c_{3} \frac{x_{k+1}}{\exp \left(x_{k}\right)}
$$

for all $k \in \mathbb{N}_{0}$.

Proof. Let $k \in \mathbb{N}_{0}$ and $Q \subset R\left(x_{k}\right)$ be a square with sides of length $r$. One can check that $f$ is conformal in a neighbourhood of $Q$. Using (14) we obtain

$$
N\left(f_{\mid Q}\right)<2 \operatorname{diam}(Q)=\frac{2 \sqrt{2}}{16 \sqrt{2}}=\frac{1}{8}
$$

and thus

$$
L\left(f_{\mid Q}\right) \leq 1+8 N\left(f_{\mid Q}\right)<2
$$

by Lemma 2.1. Therefore,

$$
\begin{aligned}
\operatorname{diam}(f(Q)) & \leq \sup \left\{\left|f^{\prime}(z)\right|: z \in Q\right\} \operatorname{diam}(Q) \\
& \leq L\left(f_{\mid Q}\right)\left|f^{\prime}\left(z_{0}\right)\right| \sqrt{2} r \\
& <2 \sqrt{2}\left|f^{\prime}\left(z_{0}\right)\right| r
\end{aligned}
$$


and

$$
\begin{aligned}
\operatorname{meas}(f(Q)) & \geq \inf \left\{\left|f^{\prime}(z)\right|^{2}: z \in Q\right\} \operatorname{meas}(Q) \\
& \geq \frac{\left|f^{\prime}\left(z_{0}\right)\right|^{2} r^{2}}{L\left(f_{\mid Q}\right)^{2}} \\
& >\frac{1}{4}\left|f^{\prime}\left(z_{0}\right)\right|^{2} r^{2} .
\end{aligned}
$$

Note from (13) that

$$
\left|f^{\prime}\left(z_{0}\right)\right|>\frac{1}{4} \exp \left(\left|\operatorname{Re} z_{0}\right|\right)>\frac{1}{4} \exp \left(x_{k}\right)
$$

for all $z_{0} \in Q$. Lemma 2.3, (12), (16), (17), (18), and (19) now show that

$$
\begin{aligned}
& \operatorname{dens}\left(\bigcup\left\{Q_{r} \in G_{r}: Q_{r} \subset f(Q) \cap R\left(x_{k+1}\right)\right\}, f(Q)\right) \\
\geq & \frac{\operatorname{meas}\left(\bigcup\left\{Q_{r} \in G_{r}: Q_{r} \cap f(Q) \neq \emptyset\right\}\right)}{\operatorname{meas}(f(Q))} \\
& -\frac{\operatorname{meas}\left(\bigcup\left\{Q_{r} \in G_{r}: Q_{r} \subset f(Q) \cap\left(\mathbb{C} \backslash R\left(x_{k+1}\right)\right)\right\}\right)}{\operatorname{meas}(f(Q))} \\
& -\frac{\operatorname{meas}\left(\bigcup\left\{Q_{r} \in G_{r}: Q_{r} \cap\left(\partial f(Q) \cup\left(\partial R\left(x_{k+1}\right) \cap f(Q)\right)\right) \neq \emptyset\right\}\right)}{\operatorname{meas}(f(Q))} \\
\geq & 1-\frac{2 x_{k+1} \operatorname{diam}(f(Q))}{\operatorname{meas}(f(Q))}-\frac{\left(12+c_{2} L\left(f_{\mid Q}\right)\left|f^{\prime}\left(z_{0}\right)\right|\right) r^{2}}{\operatorname{meas}(f(Q))} \\
\geq & 1-\frac{4\left(2 x_{k+1} 2 \sqrt{2}\left|f^{\prime}\left(z_{0}\right)\right| r+\left(12+2 c_{2}\left|f^{\prime}\left(z_{0}\right)\right|\right) r^{2}\right)}{\left|f^{\prime}\left(z_{0}\right)\right|^{2} r^{2}} \\
\geq & 1-\left(\frac{4^{2}\left(2 x_{k+1} 2 \sqrt{2}\right)}{r \exp \left(x_{k}\right)}+\frac{4^{2}\left(12+2 c_{2}\left|f^{\prime}\left(z_{0}\right)\right|\right)}{\left|f^{\prime}\left(z_{0}\right)\right| \exp \left(x_{k}\right)}\right) \\
= & 1-c_{3} \frac{x_{k+1}}{\exp \left(x_{k}\right)}
\end{aligned}
$$

with a suitable constant $c_{3}$. This completes the proof of Lemma 4.1.

Theorem 4.2. There are constants $c>0$ and $x^{*}>0$ with

$$
\operatorname{dens}\left(I(f), Q^{*}\right)>1-\frac{c}{\exp (x / 2)}
$$

for all $x \geq x^{*}$, and all squares $Q^{*}$ in $R(x)$ with sides of length $r:=1 / 16 \sqrt{2}$.

Remark. Note that the density above is independent of the distance of $Q^{*}$ to the real axis; it only depends on the distance to the imaginary axis.

Proof. Choose $x^{*}>4+2 c_{2}$ so that the conclusion of Lemma 4.1 holds. Let $x \geq x^{*}$ and $Q^{*}$ be a square in $R(x)$ with sides of length $r$. Define the sequence $\left(x_{k}\right)_{k \in \mathbb{N}_{0}}$ as in Lemma 4.1 and

$$
\operatorname{pack}\left(f^{k}(F)\right):=\left\{Q_{r} \in G_{r}: Q_{r} \subset f^{k}(F) \cap R\left(x_{k}\right)\right\}
$$

for all $F \subset Q^{*} \subset R(x)$ and all $k \in \mathbb{N}$. We construct a sequence $\left(E_{k}\right)_{k \in N_{0}}$ as follows. We define $E_{0}:=\left\{Q^{*}\right\}$ as

$$
E_{k}:=\left\{G_{k} \subset Q^{*}: G_{k} \subset F_{k-1} \in E_{k-1} \text { and } f^{k}\left(G_{k}\right) \in \operatorname{pack}\left(f^{k}\left(F_{k-1}\right)\right)\right\}
$$


and $l_{k}:=\# E_{k}$ for all $k \in \mathbb{N}$. As in Definition 3.1 we denote the elements of $E_{k}$ by $F_{k, i}$ where $i \in\left\{1, \ldots, l_{k}\right\}$ which means in particular $F_{0,1}=Q^{*}$. Then Lemma 4.1 yields that

$$
\operatorname{dens}\left(\bigcup \operatorname{pack}\left(f^{k+1}\left(F_{k, i}\right)\right), f^{k+1}\left(F_{k, i}\right)\right) \geq 1-c_{3} \frac{x_{k+1}}{\exp \left(x_{k}\right)}
$$

for $k \in \mathbb{N}_{0}, i \in\left\{1, \ldots, l_{k}\right\}$.

It is easy to see that $\left(E_{k}\right)_{k \in N_{0}}$ satisfies (8), (9) and (10) of the nesting conditions in Definition 3.1. We next verify condition (11).

Let $i \in\left\{1, \ldots, l_{k}\right\}$ and $h: f^{k+1}\left(F_{k, i}\right) \rightarrow Q^{*}$ be a branch of the inverse function of $f^{k+1}$. Using (13) and (14) it follows from the Distortion Lemma 2.2 that

$$
L(h):=L\left(h_{\mid\left(f^{k+1}\left(F_{k, i}\right)\right)}\right)<c_{1} .
$$

Note from (2), (20) and (21) that

$$
\begin{aligned}
\operatorname{dens} & \left(\bigcup_{j=1}^{l_{k+1}} F_{k+1, j}, F_{k, i}\right)=1-\operatorname{dens}\left(F_{k, i} \backslash \bigcup_{j=1}^{l_{k+1}} F_{k+1, j}, F_{k, i}\right) \\
= & 1-\operatorname{dens}\left(h\left(f^{k+1}\left(F_{k, i} \backslash \bigcup_{j=1}^{l_{k+1}} F_{k+1, j}\right)\right), h\left(f^{k+1}\left(F_{k, i}\right)\right)\right) \\
& \geq 1-L(h)^{2} \operatorname{dens}\left(f^{k+1}\left(F_{k, i}\right) \backslash \bigcup \operatorname{pack}\left(f^{k+1}\left(F_{k, i}\right)\right), f^{k+1}\left(F_{k, i}\right)\right) \\
& >1-c_{1}^{2}\left(1-\operatorname{dens}\left(\bigcup \operatorname{pack}\left(f^{k+1}\left(F_{k, i}\right)\right), f^{k+1}\left(F_{k, i}\right)\right)\right) \\
& \geq 1-c_{4} \frac{x_{k+1}}{\exp \left(x_{k}\right)},
\end{aligned}
$$

where $c_{4}=c_{1}^{2} c_{3}$. If $x^{*}$ is large enough, then

$$
1-c_{4} \frac{x_{k+1}}{\exp \left(x_{k}\right)}=1-c_{4} \frac{2}{\exp \left(\exp ^{k}(x / 2)\right)}>\frac{1}{2}
$$

for all $k \in \mathbb{N}_{0}$. Hence nesting condition (11) is satisfied by setting

$$
\delta_{k}:=1-c_{4} x_{k+1} / \exp \left(x_{k}\right),
$$

for all $k \in \mathbb{N}_{0}$. With $E:=\bigcap_{k=1}^{\infty}\left(\bigcup_{i=1}^{l_{k}} F_{k, i}\right)$, we obtain

$$
\operatorname{dens}\left(I(f), Q^{*}\right) \geq \operatorname{dens}\left(E, Q^{*}\right) \geq \prod_{k=0}^{\infty} \delta_{k} .
$$

One can show by elementary calculation that the infinite product on the righthand side is convergent. In fact, using

$$
\exp ^{k+1}(x) \geq \exp (k) \exp (x)
$$

for all $k \in \mathbb{N}_{0}$ and all $x \in \mathbb{R}$ and

$$
\log (1-x)>-2 x
$$

for all $x \in\left[0, \frac{1}{2}\right]$, we obtain

$$
\log \left(\prod_{k=0}^{\infty} \delta_{k}\right)=\sum_{k=0}^{\infty} \log \left(1-\frac{2 c_{4}}{\exp \left(\exp ^{k}(x / 2)\right)}\right)>-\frac{8 c_{4}}{\exp (x / 2)}
$$


It follows that

$$
\operatorname{dens}\left(I(f), Q^{*}\right)>\exp \left(-\frac{8 c_{4}}{\exp (x / 2)}\right) \geq 1-\frac{8 c_{4}}{\exp (x / 2)} .
$$

Setting $c:=8 c_{4}$ completes the proof of Theorem 4.2.

Proof of Theorem 1.2. Let $S^{\prime}:=\{z \in \mathbb{C}: 0 \leq \operatorname{Im} z \leq 2 \pi$ and Re $z \geq 0\}$. Let $r:=1 / 16 \sqrt{2}$ and $l_{0} \in \mathbb{N}$ with $l_{0}>2 \pi / r$. Define

$$
Q_{k}^{l}:=\{z \in \mathbb{C}: l r \leq \operatorname{Im} z \leq(l+1) r \text { and } k r \leq \operatorname{Re} z \leq(k+1) r\}
$$

for all $k \in \mathbb{N}_{0}$ and $0 \leq l \leq l_{0}$. Then

$$
S^{\prime} \subset \bigcup_{k=0}^{\infty} \bigcup_{l=0}^{l_{0}} Q_{k}^{l} .
$$

Fix $x^{*}$ as in Lemma 4.2. There is $k_{0} \in \mathbb{N}$ with $Q_{k}^{l} \subset R\left(x^{*}\right)$ for all $k \geq k_{0}$ and all $0 \leq l \leq l_{0}$. It follows from Theorem 4.2 that

$$
\operatorname{dens}\left(I(f), Q_{k}^{l}\right)>1-\frac{c}{\exp \left(\frac{r k}{2}\right)}
$$

for all $k \geq k_{0}$. We obtain

$$
\begin{aligned}
\operatorname{meas}\left(S^{\prime} \backslash I(f)\right) & \leq \operatorname{meas}\left(\left(\bigcup_{k \in \mathbb{N}_{0}} \bigcup_{l=0}^{l_{0}} Q_{k}^{l}\right) \backslash I(f)\right) \\
& \leq \sum_{k=0}^{\infty} \sum_{l=0}^{l_{0}} \operatorname{meas}\left(Q_{k}^{l} \backslash I(f)\right) \\
& =\sum_{k=0}^{\infty} \sum_{l=0}^{l_{0}}\left(1-\operatorname{dens}\left(I(f), Q_{k}^{l}\right)\right) \operatorname{meas}\left(Q_{k}^{l}\right) \\
& \leq r^{2}\left(\sum_{k=0}^{k_{0}-1} \sum_{l=0}^{l_{0}} 1+\sum_{k=k_{0}}^{\infty} \sum_{l=0}^{l_{0}} \frac{c}{\exp \left(\frac{r k}{2}\right)}\right) \\
& =\left(l_{0}+1\right) r^{2}\left(k_{0}+c \exp \left(-\frac{r k_{0}}{2}\right) \frac{1}{1-\exp \left(-\frac{r}{2}\right)}\right) \\
& <\infty .
\end{aligned}
$$

Theorem 1.2 follows directly with the $2 \pi i$-periodicity of the hyperbolic sine and the symmetry with respect to the imaginary axis.

Remark. A computation shows that we can take $x^{*}:=45, c_{3}:=2049, c:=65568 e^{4}$, $k_{0}:=1019$, and $l_{0}:=143$ and we can obtain 574 as an upper bound for the area of $S \backslash I(f)$. But we have made no effort to obtain sharp bounds.

\section{ACKNowledgments}

The author wants to thank the referee and David Drasin for helpful comments, and in particular Walter Bergweiler for all his support. 


\section{REFERENCES}

1. A. F. Beardon, Iteration of rational functions, Springer, 1991 MR1128089 (92j:30026)

2. W. Bergweiler, Iteration of meromorphic functions, Bull. Amer. Math. Soc. 29 (1993), 151-188 MR1216719 (94c:30033)

3. P. Domínguez, Connectedness properties of Julia sets of transcendental entire functions, Complex Variables Theory Appl. 32 (1997), 199-215 MR1457686 (98f:30027)

4. P. Domínguez and G. Sienra, A study of the dynamics of $\lambda \sin z$, Internat. J. Bifur. Chaos Appl. Sci. Engrg. 12 (2002), 2869-2883 MR1956410 (2003m:37060)

5. A. E. Eremenko and M. Yu. Lyubich, Dynamical properties of some classes of entire functions, Ann. Inst. Fourier, Grenoble 42 (1992), 989-1019 MR1196102 (93k:30034)

6. P. Fatou, Sur l'itération des fonctions transcendantes entières, Acta Math. 47 (1926), 337-370 MR1555220

7. B. Karpińska, On the accessible points in the Julia sets of some entire functions, Fund. Math. 180 (2003), 89-98 MR2064852 (2005h:37098)

8. B. Karpińska, Area and Hausdorff dimension of the set of accessible points in the Julia sets of $\lambda e^{z}$ and $\lambda \sin z$, Fund. Math. 159 (1999), 269-287 MR1680622 (2000b:37042)

9. C. McMullen, Area and Hausdorff dimension of Julia sets of entire functions, Trans. Amer. Math. Soc. 300 (1987), 329-342 MR871679 (88a:30057)

10. J. Milnor, Dynamics in one complex variable, Second Edition, Vieweg, 2000 MR1721240 (2002i:37057)

11. G. Rottenfußer and D. Schleicher, Escaping points of the cosine family, ArXiv math.DS/0403012 (2004)

12. D. Schleicher, The dynamical fine structure of iterated cosine maps and a dimension paradox, Duke Math. J., 136 (2007), 343-356. MR2286634

13. H. Schubert, Über das Maß der Fatoumenge trigonometrischer Funktionen, Diploma thesis, Kiel University, 2003

14. N. Steinmetz, Rational Iteration, De Gruyter, 1993 MR1224235 (94h:30035)

15. H. Töpfer, Über die Iteration der ganzen transzendenten Funktionen, insbesondere von $\sin z$ und $\cos z$, Math. Ann. 117 (1939), 65-84 MR0001293 (1:211e)

Department of Mathematics, Kiel University, 24098 Kiel, Germany

E-mail address: schubert@math.uni-kiel.de 